\title{
Empirical Solar Abundance Scalings of Supernova Gamma-Process Isotopes
}

\section{T. Hayakawa*i, T. Shizuma}

Kansai Photon Science Institute, Japan Atomic Energy Agency, Kizu, Kyoto 619-0215, Japan. E-mail: hayakawa.takehitoejaea.go.jp

\section{N. Iwamoto}

Nuclear Data Center, Japan Atomic Energy Agency, Tokai, Ibaraki 319-1195, Japan.

\section{T. Kajino}

National Astronomical Observatory, Osawa, Mitaka, Tokyo 181-8588, Japan.

\section{H. Umeda}

Department of Astronomy, Graduate School of Science, University of Tokyo, Bunkyo-ku, Tokyo 113-0033, Japan.

\section{K. Nomoto}

Department of Astronomy \& Research Center for the Early Universe, School of Science, University of Tokyo, Bunkyo-ku, Tokyo 113-0033, Japan.

Institute for the Physics and Mathematics of the Universe, University of Tokyo, Kashiwa, Chiba 277-8568, Japan.

\begin{abstract}
Analyzing the solar system abundances, we have found two empirical scaling laws concerning the $p$ - and $s$-nuclei with the same atomic number. These scalings are evidence that most probable origin of $27 p$-nuclei is the supernova $\gamma$-process. We have calculated the abundance ratios by the $\gamma$-process nucleosynthesis based on core-collapse supernova explosion models under various astrophysical conditions. The results show that the scalings hold for materials produced by individual $\gamma$-processes independent of the astrophysical conditions assumed. We also discuss the origin of the other $8 p$-nuclei.
\end{abstract}

10th Symposium on Nuclei in the Cosmos

July 27 - August 12008

Mackinac Island, Michigan, USA

\footnotetext{
* Speaker.

${ }^{\dagger}$ This work has been supported in part by Grants-in-Aid for Scientific Research (18340071).
} 


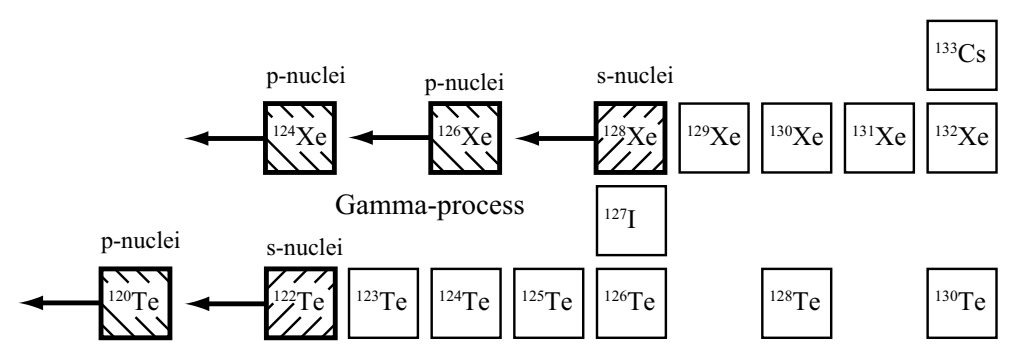

Figure 1: A partial nuclear chart and nucleosynthesis flow around Te and Xe.

\section{Introduction}

The solar system was formed from the interstellar medium (ISM), which composition has been provided from many different stellar nucleosynthesis episodes and the solar abundances have provided crucial evidence of astrophysical origin of materials. Isotopes heavier than the iron group are synthesized by $s$ - and $r$-processes but the origin of 35 neutron-deficient rare isotopes, $p$-nuclei, have been discussed over the last 50 years. Woosley and Howard found the anti-correlation between the photodisintegration reaction rates and the solar abundances of the $p$-nuclei [1], which is the first evidence that the $p$-nuclei are dominantly synthesized by photodisintegration reactions in supernova explosions ( $\gamma$-process or $p$-process) [1, 2, 3, 4]. Recently, we found two empirical scalings for the $p$ - and $s$-nuclei with the same atomic number, which are the second evidence that most probable origin of $27 p$-nuclei is supernova $\gamma$-process, and a novel concept of "universality of the $\gamma$-process" [5, 6, 7]. We also have proposed a nuclear cosmochronometer for supernova neutrino-process based on these scalings [8].

\section{Empirical solar abundance scaling laws and origin of $27 p$-nuclei}

Figure 1 shows a partial nuclear chart around Te and Xe. In this region, ${ }^{124,126} \mathrm{Xe}$ and ${ }^{120} \mathrm{Te}$ are the $p$-nuclei and ${ }^{128} \mathrm{Xe}$ and ${ }^{122} \mathrm{Te}$ are pure $s$-nuclei which are not produced by the $r$-process. The 1st and 2nd $p$-nuclei are lighter than the $s$-nucleus by two and four neutrons, respectively. Taking the abundance ratios of the $s$-nucleus to the $p$-nucleus, $\mathrm{N}_{\odot}(\mathrm{s}) / \mathrm{N}_{\odot}(p)$, where $\mathrm{N}_{\odot}(s)$ and $\mathrm{N}_{\odot}(p)$ are the solar isotope abundances of the $s$ - and $p$-nuclei, respectively. We reported the first scaling that $\mathrm{N}_{\odot}(s) / \mathrm{N}_{\odot}(p)$ ratios are almost constant over a wide region of atomic number. Most ratios are centered around an average value of 23 within a factor of 2 as showin in Fig. 2 [7]. We found another empirical scaling between two $p$-nuclei with the same atomic number. The ratios are almost constant over a wide range of the atomic number. The fact that the scalings appear in the solar abundances leads to a concept of the universality of the $\gamma$-process that the scalings hold for materials produced by individual supernova $\gamma$-process. We presented the basis of the universality of the $\gamma$-process in model calculations of core-collapse supernovae [9] under various astrophysical conditions such as, metallicity, progenitor mass, and explosion energy [7]. The results showed that the scalings hold for individual nucleosyntheses independent of the astrophysical conditions assumed and we found that the universality originated from the three mechanisms [7]. 


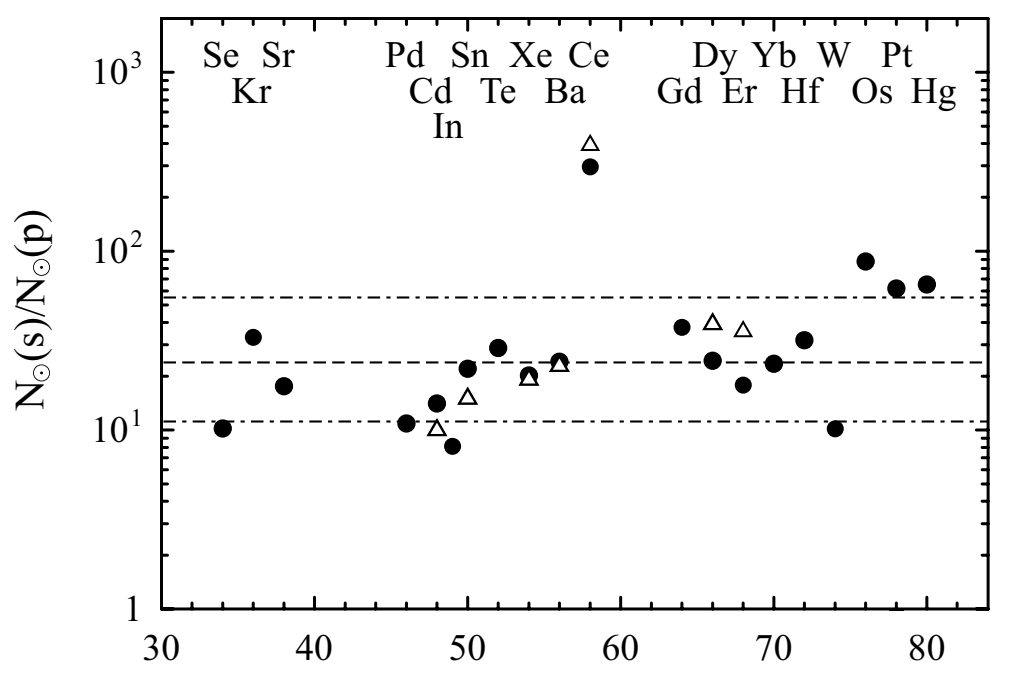

Atomic Number Z

Figure 2: The solar abundance ratios of $p$ - and $s$-nuclei with the same atomic number. The filled circles show the ratios of the $s$-nuclei to 1 st $p$-nuclei. The open triangles show the ratios of the $s$-nuclei to 2 nd p-nuclei.

\section{Origin of $8 p$-nuclei}

The two scalings are evidence that $27 p$-nuclei are dominantly produced by the $\gamma$-process. A new generation of $\gamma$-ray sources of a laser Compton scattering (LCS) $\gamma$-rays has been developed and widely used for studies of the nuclear astrophysics [11, 12, 13, 14]. These $\gamma$-ray sources are powerful tools for the study the photo-induced reactions because of their sharp energy edge and tunable energy. There are $35 p$-nuclei and the origin of the other $8 p$-nuclei, ${ }^{92,94} \mathrm{Mo},{ }^{96,98} \mathrm{Ru}$, ${ }^{144} \mathrm{Sm},{ }^{115} \mathrm{Sn},{ }^{138} \mathrm{La}$, and ${ }^{180} \mathrm{Ta}$, has remained as an open question [7]. Here we discuss the origin of these $8 p$-nuclei. Figure 3 shows an abundance distribution of the $p$-and $s$-nuclei of the members of the scalings. The correlation between the $p$ - and $s$-nuclei can be observed in this figure: the abundance pattern of the $p$-nuclei shows a tendency similar to that of the $s$-nuclei. This is consistent with the scaling that the $\mathrm{N}_{\odot}(s) / \mathrm{N}_{\odot}(p)$ ratios are almost constant.

Burbidge $e t$ al. pointed out that the mass distribution of the $p$-nuclei show two peaks near two neutron magic numbers of $\mathrm{N}=50,82$ and the $p$-nuclei may be synthesized by $(\mathrm{p}, \gamma)$ and $(\gamma, \mathrm{n})$ reactions [10]. The abundance pattern around Mo region is similar to that of Sm (see Fig. 3). The abundances of ${ }^{92,94} \mathrm{Mo}$ is larger than those of lighter $p$-isotopes, ${ }^{84} \mathrm{Sr}$ and ${ }^{78} \mathrm{Kr}$, and the abundance of ${ }^{144} \mathrm{Sm}$ is also larger than those of lighter $p$-nuclei. In contrast, the abundances of the seed $s$ nuclei, ${ }^{96} \mathrm{Mo},{ }^{100} \mathrm{Ru}$ and ${ }^{148} \mathrm{Sm}$, are consistent with those of the same mass region: namely, their abundances are lower than those of $s$-nuclei in the lighter mass regions beyond the neutron magic numbers. This fact suggests a possibility that these 5 -nuclei, ${ }^{92,94} \mathrm{Mo},{ }^{96,98} \mathrm{Ru}$, and ${ }^{144} \mathrm{Sm}$ might be synthesized mainly from $s$-nuclei located in lighter mass regions beyond the neutron magic number by particle-induced reactions such as $(\mathrm{p}, \gamma),(\alpha, \gamma)$ or $(\mathrm{n}, \gamma)$ reaction. Recently, the proton and 
alpha-induced reaction experiments have been carried out up to $A \sim 120$ [15, 16, 17]. However, the reaction rates in a heavy mass region of $A \sim 140$ have not been measured. These rates are of importance for understanding the origin of the $p$-nuclei near both the neutron magic numbers of $N$ $=50$ and 82 [7].

Recent progress of the meteorite science give crucial hints about the origin of Mo and Sm. Yin et al. measured isotopic fractions of Mo in primitive meteorites with high accuracy and the isotopic fractions of the $p$-nucleus ${ }^{94} \mathrm{Mo}$ and the $r$-nucleus ${ }^{100} \mathrm{Mo}$ show different anomalies in comparison with the solar abundances [18]. This result suggests that the nucleosynthesis site of ${ }^{94} \mathrm{Mo}$ is not correlated with the $r$-process site. Isotopic anomalies of $\mathrm{Sm}$ in primitive meteorites were also measured and the results showed that nucleosynthesis sites supplying the $p$ - and $r$-isotopes were disconnected [19]. It is of importance measurements of the isotope abundances of both the elements, Sm and Mo, in individual meteoritic samples, which can answer the question whether the nucleosynthesis sites of Sm and Mo are same or not.

As the origin of ${ }^{115} \mathrm{Sn}$, the $\gamma$-, $s$ - and $r$-processes were proposed but it has been an open question [20] ${ }^{138} \mathrm{La}$ and ${ }^{180} \mathrm{Ta}$ are odd-odd nuclei and their isotope ratios are small, $0.090 \%$ (for ${ }^{138} \mathrm{La}$ ) and $0.012 \%\left({ }^{180} \mathrm{Ta}\right)$. As the origin of these $2 p$-nuclei, the cosmic-ray process [21] and the $v$-process [22] were proposed. Recent studies of the $v$-process can explain the solar abundance of ${ }^{138} \mathrm{La}$ but they overproduce that of ${ }^{180} \mathrm{Ta}[23,24]$. A study of primitive meteorites provides a hint about the origin of ${ }^{138} \mathrm{La}$. Shen and Lee reported the isotope abundance anomalies of several elements as $\mathrm{Ti}$ and $\mathrm{La}$ in Al-Ca-rich inclusions, which is considered to originated from the early solar nebula. The abundance ratios of ${ }^{138} \mathrm{La} /{ }^{139} \mathrm{La}$ is correlated with that of ${ }^{50} \mathrm{Ti} /{ }^{48} \mathrm{Ti}[25]$. The reason why ${ }^{138} \mathrm{La}$ is correlated with ${ }^{50} \mathrm{Ti}$ has been an open question. The measurements of both isotopic fractions of $\mathrm{La}$ and Ta will provide a crucial hint for origin of ${ }^{138} \mathrm{La}$ and ${ }^{180} \mathrm{Ta}$.

\section{Conclusion}

\section{References}

[1] S.E. Woosley, W.M. Howard, Astrophys. J. Suppl. 36, 285 (1978).

[2] M. Arnould, Astron. Astrophys. 46, 117 (1976).

[3] M. Rayet, M. Arnould, M. Hashimoto, N. Prantzos, K. Nomoto, Astron. Astrophys. 298, 517 (1995).

[4] W. Rapp, et al., Astrophys. J., 474, 653 (2006).

[5] T. Hayakawa, N. Iwamoto, T. Shizuma, T. Kajino, H. Umeda, K. Nomoto, Phys. Rev. Lett. 93, 161102 (2004).

[6] T. Hayakawa, N. Iwamoto, T. Kajino, T. Shizuma, H. Umeda, K. Nomoto, Astrophys. J., 648, L47 (2006).

[7] T. Hayakawa, et al., Astrophys. J. 685, 1089 (2008).

[8] T. Hayakawa, T. Shizuma, T. Kajino, K. Ogawa, H. Nakada, Phys. Rev. C 77, 065802 (2008).

[9] K. Nomoto, et al., in Stellar Collapse, ed. C.L. Fryer (Astrophysics and Space Science: Kluwer), 277 (astro-ph/0308136) (2004). 


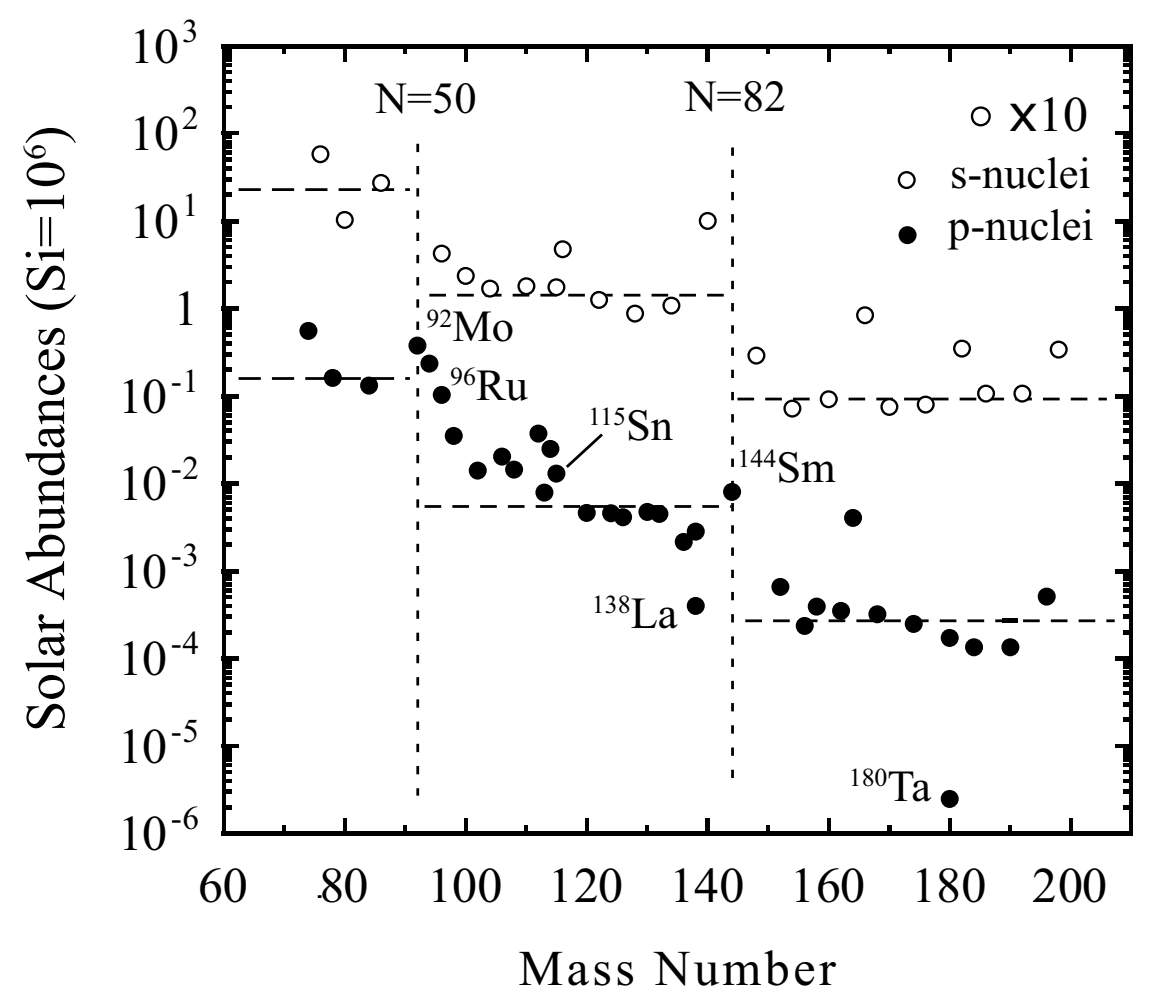

Figure 3: The solar abundances of $p$ - and $s$-nuclei in the pairs of the scalings.

[10] E.M. Burbidge, G.R. Burbidge, W.A. Fowler, F. Hoyle, Rev. Mod. Phys. 29, 548 (1957).

[11] P. Mohr, et al., Phys. Rev. C 69, 032801(R) (2004).

[12] T. Shizuma, et al., Phys. Rev. C 72, 025808 (2005).

[13] T. Hayakawa, et al., Phys. Rev. C 74, 065802 (2006).

[14] T. Hayakawa, et al., Phys. Rev. C 77, 068801 (2008).

[15] N. Özkan, et al., Nucl. Phys. A 710, 469 (2002).

[16] G.G. Kiss, et al., Eur. Phys. J. A 27, 197 (2006).

[17] Gy. Gyürky, Gy., et al., Phys. Rev. C 74, 025805 (2006).

[18] Q. Yin, S.B. Jacobsen, K. Yamashita, Nature 415, 881 (2002).

[19] R. Andreasen, \& M. Sharma, Science, 314, 806 (2006).

[20] Zs. Németh, et al., Astrophys. J., 426, 357 (1994).

[21] J. Audouze, 1970, Astronom. \& Astrophys., 8, 436 (1970).

[22] S. E. Woosley, D. H. Hartmann, R. D. Hoffman, W. C. Haxton, Astrophys. J. 356, 272 (1990).

[23] A. Heger, et al., Phys. Lett. B, 606, 258 (2005).

[24] A. Byelikov, et al., Phys. Rev. Lett. 98, 082501 (2007).

[25] J.J. Shen, \& T. Lee, Astrophys. J. 596, L109 (2003). 